\title{
Actual Conversion Rate from Total Mastectomy to Breast Conservation after Neoadjuvant Chemotherapy for Stages II-III Breast Cancer Patients
}

\author{
Hyejin Mo ${ }^{1}$, Yumi Kim ${ }^{1}$, Jiyoung Rhu' ${ }^{1}$, Kyung-Hun Lee ${ }^{2,3}$, Tae-Yong Kim ${ }^{2,3}$, Seock-Ah Im $^{2,3}$, Eun-Shin Lee ${ }^{1}$, \\ Han-Byoel Lee ${ }^{1}$, Hyeong-Gon Moon ${ }^{1,3}$, Dong-Young Noh ${ }^{1,3}$, Wonshik Han ${ }^{1,3}$ \\ Departments of ${ }^{1}$ Surgery and ${ }^{2}$ Internal Medicine, Seoul National University College of Medicine, Seoul; ${ }^{3}$ Cancer Research Institute, Seoul National \\ University, Seoul, Korea
}

\begin{abstract}
Purpose: Neoadjuvant chemotherapy (NCT) is a treatment modality that increases the breast-conserving rate in breast cancer. This prospective study was performed to evaluate the actual breast-conserving rate using NCT in a clinical setting in a single institution. Methods: Between 2014 and 2015, 265 patients who were scheduled to receive NCT and surgery were enrolled in this study. Patients were classified into three groups based on the immunohistochemical results of estrogen receptor (ER)/progesterone receptor (PR) and human epidermal growth factor receptor 2 (HER2): ER or PR positive (luminal), ER/PR negative and HER2 positive (HER2+), and triple-negative breast cancer (TNBC). Before starting and immediately after completing NCT, a surgeon decided if breast-conserving surgery (BCS) or total mastectomy (TM) should be performed. We analyzed the rate of type of surgery performed. Results: Before administering NCT, 107 patients (40.4\%) and 158 patients (59.6\%) were candidates for BCS and TM, respectively. Of the 158 patients, 61 were eligible for BCS after chemotherapy, with a conversion rate of $38.6 \%$. NCT increased the BCS eligible rate from $40.4 \%$ to $62.6 \%$. Of the 61 patients, 53 chose to undergo BCS, and BCS was successful in $46(86.8 \%)$. Of the 107 BCS candidates at baseline, 100 patents finally underwent BCS $(93.5 \%)$. According to the subtype, the conversion rates were $35.4 \%, 50.0 \%$, and $40.5 \%$ for luminal, HER2+, and TNBC groups, respectively. Conclusion: NCT increased the eligibility for BCS from $40.4 \%$ to $62.6 \%$ in a clinical setting. This benefit is similar to that observed in other clinical trials.
\end{abstract}

Key Words: Breast-conserving surgery, Breast neoplasms, Neoadjuvant chemotherapy

\section{INTRODUCTION}

Neoadjuvant chemotherapy (NCT) is a widely used treatment modality for early and locally advanced breast cancer because it increases the likelihood of breast-conserving surgery (BCS) [1]. Several studies have investigated the oncologic safety and the extent to which NCT can actually convert total mastectomy (TM) candidates to BCS candidates [2]. The results implicate that NCT may improve the patients' quality of life by allowing BCS in as many patients as possible [3].

However, most of the studies showing the benefit of NCT in increasing the BCS rate were retrospective analyses conducted in patients enrolled in clinical trials that test the efficacy of NCT [4-6]. Recently, Golshan et al. [2] published the results from two prospective

Correspondence: Wonshik Han

Department of Surgery and Cancer Research Institute, Seoul National University College of Medicine, 101 Daehak-ro, Jongno-gu, Seoul 03080, Korea Tel: +82-2-2072-1958, Fax: +82-2-766-3975, E-mail: hanw@snu.ac.kr Received: Oct 8, 2017 Revised: Oct 30, 2017 Accepted: Nov 26, 2017 studies on the impact of NCT on BCS eligibility and BCS rate in triple-negative breast cancer (TNBC) and human epidermal growth factor receptor 2 (HER2)-positive breast cancer as companion studies to the CALGB40603 and 40601 trials [7]. They showed that NCT results in $14 \%$ and $23 \%$ absolute BCS eligibility increase in TNBC and HER2-positive patients, respectively.

In this study, by analyzing patients undergoing NCT and surgery in a single institution, the effects of NCT on the conversion from TM to BCS were prospectively examined in a routine clinical setting. Moreover, we attempted to determine the difference in the conversion rate based on the intrinsic subtype of breast cancer.

\section{METHODS}

Patients with clinical stage II or III primary breast cancer who were scheduled for NCT before surgery during the study period were included. Patients who did not complete the full cycles of NCT due to 
Table 1. Neoadjuvant chemotherapy regimens used in the study

\begin{tabular}{lc}
\hline Chemotherapy regimen & $\begin{array}{c}\text { No. of } \\
\text { patients }\end{array}$ \\
\hline Doxorubicin/cyclophosphamide \#4 $\rightarrow$ docetaxel \#4 & 188 \\
Doxorubicin/cyclophosphamide \#4 $\rightarrow$ docetaxel/herceptin \#4 & 44 \\
Doxorubicin/cyclophosphamide \#4 $\rightarrow$ paclitaxel \#4 & 1 \\
Doxorubicin/cyclophosphamide \#4 $\rightarrow$ paclitaxel/herceptin \#4 & 2 \\
$\begin{array}{l}\text { Docetaxel/doxorubicin \#6 } \\
\begin{array}{l}\text { Docetaxel+herceptin \#4 } \rightarrow \text { fluorouracil/epirubicin/cyclophos- } \\
\text { phamide+herceptin \#4 }\end{array}\end{array}$ \\
\hline
\end{tabular}

toxicity or disease progression were included in the analysis $(n=12)$. Finally, 265 patients were enrolled. Table 1 shows the regimens used for patients who completed NCT as scheduled. The most common regimen (92.9\%) was four cycles of doxorubicin/cyclophosphamide, followed by four cycles of taxane with or without trastuzumab.

Mammography, breast ultrasonography, and clinical breast examination were performed in all patients before starting NCT. Mammography, breast ultrasonography, and breast magnetic resonance imaging (MRI) were performed immediately after completing NCT. A treating surgeon (W.H.) assessed the ineligibility for BCS before and after the initiation of NCT based on the following five criteria: (1) large tumor size compared to breast size; (2) diffuse calcification; (3) multicentric tumor; (4) nipple invasion or tumors located close to the nipple; and (5) poor cosmetic result expected with BCS. BCS or TM was performed after completing all the cycles of NCT, following the informed discussion between the treating surgeon and patients. Successful BCS was defined as the absence of the tumor cells on resection margins of lumpectomy specimen. If the resection margin was positive for tumor cells, a wider excision or TM was done according to the surgeon's discretion.

Patients were classified into the following three types based on the immunohistochemical results of estrogen receptor(ER)/progesterone receptor (PR) and HER2: ER or PR positive (luminal), ER/PR negative and HER2 positive (HER2+), and TNBC.

The chi-square test, Fisher exact test, and the linear-by-linear association were used to analyze the factors affecting the conversion to BCS. All analyses were performed using the IBM SPSS Statistics/PC software package version 22.0 (IBM Corp., Armonk, USA). The informed consent was waived this study was approved by the Institutional Review Board of Seoul National University Hospital (IRB number: 1609-082-792).
Table 2. Clinicopathological features of BCS and TM candidates before neoadjuvant chemotherapy

\begin{tabular}{|c|c|c|}
\hline Variable & $\begin{array}{c}\text { Initial BCS candidates } \\
\text { No. (\%) }\end{array}$ & $\begin{array}{c}\text { Initial TM candidates } \\
\text { №. (\%) }\end{array}$ \\
\hline No. of patients & 107 & 158 \\
\hline Average age at diagnosis (yr) & 48.1 & 47.0 \\
\hline \multicolumn{3}{|l|}{ Clinical stage at diagnosis } \\
\hline T2NOMO & $17(15.9)$ & $15(9.5)$ \\
\hline T3NOMO & 0 & $7(4.4)$ \\
\hline T4NOMO & 0 & 0 \\
\hline $\mathrm{T} 1 \mathrm{~N}+\mathrm{M} 0$ & $18(16.8)$ & $11(7.0)$ \\
\hline $\mathrm{T} 2 \mathrm{~N}+\mathrm{M} 0$ & $72(67.3)$ & $84(53.2)$ \\
\hline T3N+M0 & 0 & $37(23.4)$ \\
\hline $\mathrm{T} 4 \mathrm{~N}+\mathrm{M} 0$ & 0 & $4(2.5)$ \\
\hline \multicolumn{3}{|l|}{ ER status } \\
\hline Negative & $47(43.9)$ & $63(39.9)$ \\
\hline Positive & $60(56.1)$ & $95(60.1)$ \\
\hline \multicolumn{3}{|l|}{ PR status } \\
\hline Negative & $64(59.8)$ & $86(54.4)$ \\
\hline Positive & $43(40.2)$ & $72(45.6)$ \\
\hline \multicolumn{3}{|l|}{ HER2 status } \\
\hline Negative & $74(69.2)$ & $113(71.5)$ \\
\hline Positive & $33(30.8)$ & $45(28.5)$ \\
\hline \multicolumn{3}{|l|}{ Subtype } \\
\hline Luminal & $60(56.1)$ & $96(60.8)$ \\
\hline HER2-positive & $16(15.0)$ & $20(12.7)$ \\
\hline TNBC & $31(29.0)$ & $42(26.6)$ \\
\hline \multicolumn{3}{|l|}{ Ki-67 index (\%) } \\
\hline$\leq 10$ & $41(38.3)$ & $65(41.1)$ \\
\hline$>10$ & $59(55.1)$ & $88(55.7)$ \\
\hline Unknown & $7(6.5)$ & $5(3.2)$ \\
\hline \multicolumn{3}{|l|}{ Histologic grade } \\
\hline Grade 1 & $4(3.7)$ & $4(2.5)$ \\
\hline Grade 2 & $54(50.5)$ & $92(58.2)$ \\
\hline Grade 3 & $48(44.9)$ & $62(39.2)$ \\
\hline Unknown & $1(0.9)$ & 0 \\
\hline
\end{tabular}

$\mathrm{BCS}=$ breast-conserving surgery; $\mathrm{TM}=$ total mastectomy; $\mathrm{ER}=$ estrogen receptor; $\mathrm{PR}=$ progesterone receptor; $\mathrm{HER} 2$ = human epidermal growth factor receptor 2; TNBC = triple-negative breast cancer.

\section{RESULTS}

Of the 265 patients, 107 (40.4\%) were BCS and 158 (59.6\%) were TM candidates before initiating NCT. Table 2 shows the patients' demographics.

After NCT, 61 of the 158 TM candidates were converted to BCS candidates, with the conversion rate of $38.6 \%$ (Figure 1). Based on the subtype, the conversion rate was $35.4 \%$ (34/96), 50.0\% (10/20), and $40.5 \%$ (17/42) among patients with the luminal, HER2+, and TNBC, respectively. The absolute increase in BCS eligibility was 22.2\% (from 


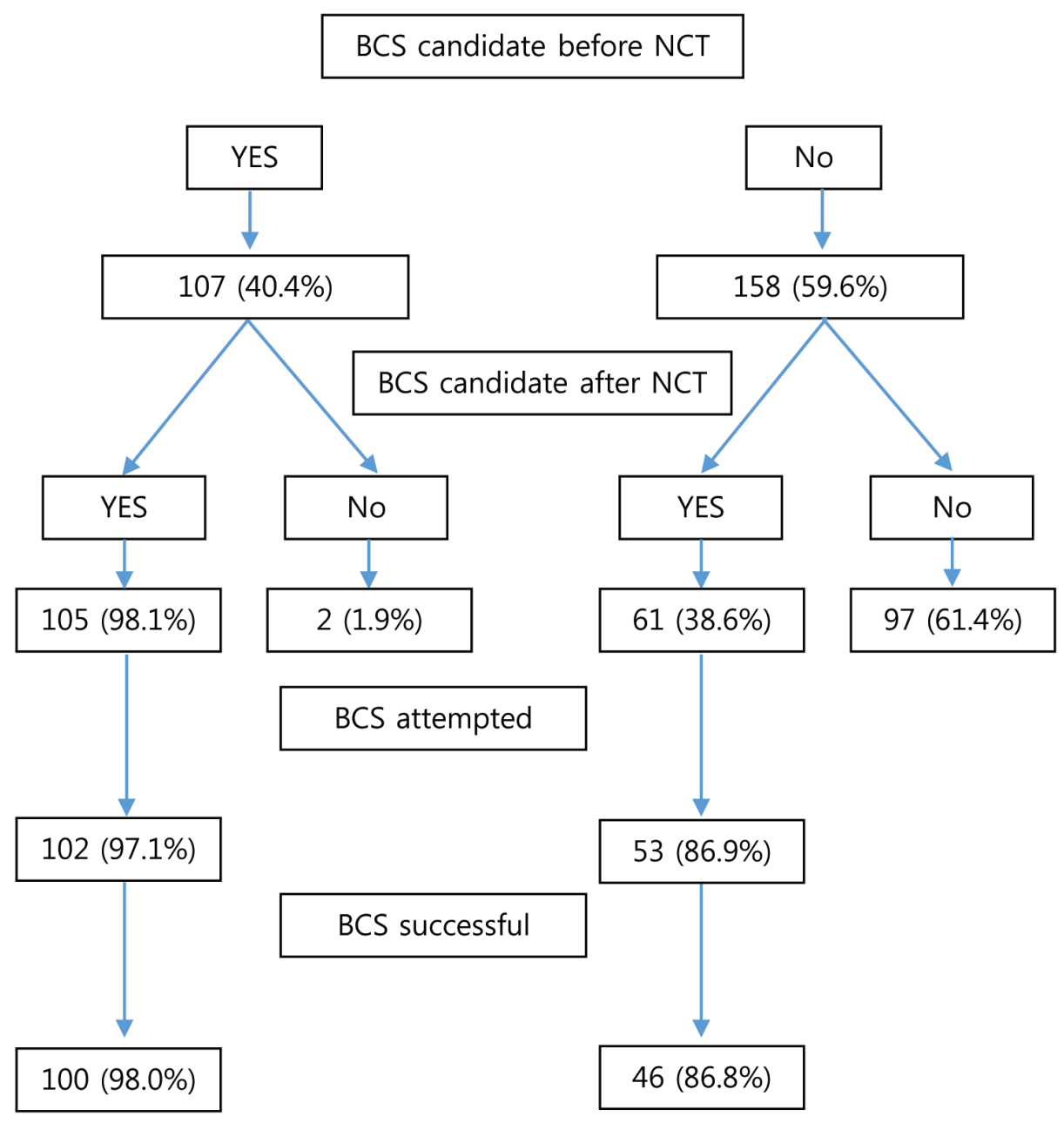

Figure 1. Summary of the effect of neoadjuvant chemotherapy (NCT) on surgery. $\mathrm{BCS}=$ breast-conserving surgery.

Table 3. Exclusion criteria for breast-conserving surgery

\begin{tabular}{lcc}
\hline Criteria & $\begin{array}{c}\text { Before NCT }(n=158) \\
\text { No. }(\%)\end{array}$ & $\begin{array}{c}\text { After NCT }(n=99) \\
\text { No. }(\%)\end{array}$ \\
\hline 1. Large size & $67(42.4)$ & $51(51.5)$ \\
2. Diffuse calcification & $4(2.5)$ & $4(4.0)$ \\
3. Multicentricity & $23(14.6)$ & $23(23.2)$ \\
4. Nipple invasion & $11(7.0)$ & $15(15.2)$ \\
5. Poor cosmesis expected & $53(33.5)$ & $6(6.1)$ \\
\hline
\end{tabular}

$\mathrm{NCT}=$ neoadjuvant chemotherapy.

$40.4 \%$ to $62.6 \%$ ). Table 3 presents the exclusion criteria for BCS. Large tumor size was the most common characteristic for exclusion for BCS.

Among the 107 patients who were originally BCS candidates, five underwent TM after NCT. Although BCS could have been attempted, three patients preferred to undergo TM. MRI after NCT showed wide tumor extent in two patients. Out of the 102 patients who underwent
BCS, including three reoperations for wider excision, the final resection margin was clear in 100 patients, and the success rate was $98.0 \%$. Two patients underwent TM.

Of the 61 patients who became candidates for BCS with NCT, TM was performed for eight patients considering their choice. As a result, 53 patients received BCS with the success rate of $86.8 \%$ (46/53). Seven patients underwent TM in this group.

The tumor size and BCS conversion rate were inversely correlated. The larger the initial tumor size before NCT, the lower was the BCS conversion rate (Figure 2).

A total of $35.4 \%, 50.0 \%$, and $40.5 \%$ of patients were converted to BCS candidates among the patients with luminal, HER2+ and TNBC subtypes, respectively ( $p=0.305$, luminal vs. HER $2+$ and TNBC) (Figure 3). 


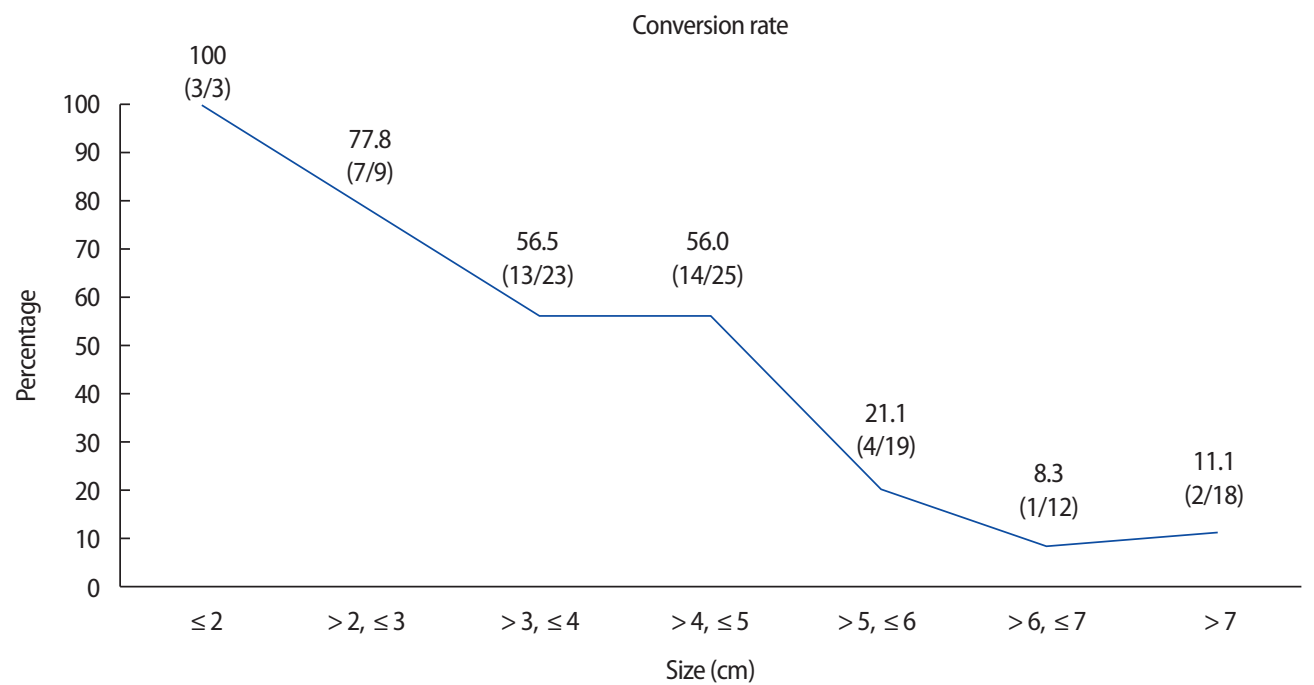

Figure 2. Correlation between tumor size and breast-conserving surgery conversion rate.

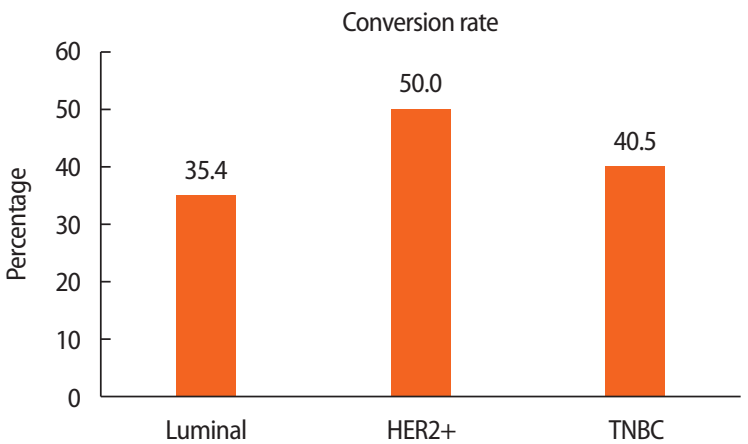

Figure 3. Breast-conserving surgery conversion rate according to breast cancer type.

HER2+=human epidermal growth factor receptor 2 positive; TNBC=triple-negative breast cancer.

\section{DISCUSSION}

NCT results in surgical downstaging of tumors and allows breast conservation in women who otherwise would have needed mastectomy. In the NSABP B-18 study, NCT was associated with a higher rate of BCS $(68 \%$ vs. $60 \%, p=0.001)$ than adjuvant chemotherapy [8]. In the EORTC 10902 trial, the breast conservation rates were 35\% and $22 \%$ in the neoadjuvant and adjuvant arm, respectively [9]. A prospective study by Golshan et al. [2] for TNBC (CALGB40603) showed a $42 \%$ conversion rate from BCS-ineligible to BCS-eligible patients, resulting in a 14\% absolute increase in BCS eligibility. In another study on HER2-positive breast cancer (CALGB40601) by the same authors, NCT increased the BCS-eligibility rate from $41 \%$ to $64 \%[2,7]$.
To the best of our knowledge, this is the first prospective study that investigated the change in BCS eligibility with NCT in a clinical setting, but not in a clinical trial. In this study, the conversion rate from BCS to TM as a result of NCT using routine chemotherapy regimens was $38.6 \%$, which is similar to that observed in other previous studies. The absolute increase in BCS eligibility was $22.2 \%$, which is superior to that noted in the CALGB40603 study for TNBC. The increase in BCS eligibility is more noteworthy because different subtypes were evaluated in our cohort, including the luminal type. The response to NCT is worse in luminal subtype breast cancer patients [10]. In the present study, the pathologic complete response rate of the luminal type group was $14.7 \%$, which is lower than that of the other two groups (27.5\%).

Safety concerns regarding BCS after NCT have been raised. The meta-analysis by Mieog et al. [6] showed that the rates of local or regional recurrences in patients who underwent neoadjuvant versus adjuvant therapy were not different when stratified based on the type of surgery. They also found no difference in locoregional recurrences for patients who were scheduled to undergo BCS and those who were downstaged to BCS candidates. Similarly, this study showed that BCS after NCT in clinical stage III patients is oncologically safe in terms of local recurrence. The rate of local recurrence-free survival was not different between the surgery-first, preplanned BCS, and downstaged BCS groups [11].

In the CALGB40603 study by Golshan et al. [2], BCS was not 
attempted in $32 \%$ of the patients who became BCS candidates. Moreover, $20 \%$ of the patients who were BCS candidates before NCT and still BCS candidates after NCT chose mastectomy rather than BCS; this finding differed from the findings of the present study. In our study, BCS was attempted in $86.9 \%$ of the patients who became BCS eligible from being TM candidates after NCT. Among the pre- and post-NCT BCS candidates, $97.1 \%$ underwent BCS. The reason for the higher rate of patients or surgeons choosing BCS in our institute than that reported in multicenter studies performed in the United States is difficult to explain. When the cases of actual BCS application instead of BCS eligibility are considered, the conversion rate was $33.5 \%$ (53/158), and the absolute increase reached $18.1 \%$ in the present study.

Our study showed that large-sized tumors are less likely to be treated with BCS with chemotherapy (Figure 2). Patients with a clinical tumor size less than or equal to $3 \mathrm{~cm}$ based on breast ultrasonography and breast MRI were classified as TM candidates for the following reasons: two patients had multiple lesions, one patient had diffuse calcification, and nine patients were expected to show poor cosmesis when attempting BCS because the lesion was relatively large compared to the size of the breast on physical examination. In addition, two of the nine patients who were expected to show poor cosmesis had a tumor in the subareolar area. The cancer subtype did not make a significant difference in this study. However, patients who discontinued NCT may not be able to fully reflect the role of chemotherapy in BCS conversion. In the analysis of patients who completed NCT, the conversion rate was $35.8 \%$ in the luminal type and $49.1 \%$ in the other type $(p=0.125)$. Conducting studies with larger sample size are necessary considering the significantly different conversion and complete pathologic response rates between the luminal type and other breast cancer types. These findings are consistent with our previous study showing that negative ER, small initial tumor size, high Ki-67 levels, and absence of in situ component were associated with a residual tumor size of $\leq 3 \mathrm{~cm}[10]$.

A limitation of our study was that determining whether a patient will be a BCS or TM candidate was not always feasible. A significant proportion of the patients had borderline possibility of BCS. In particular, judgments about poor cosmesis may be different for each surgeon; thus, this should be considered in interpreting the results.

In conclusion, NCT increased the eligibility for BCS from 40.4\% to $62.6 \%$ in our prospective study conducted in a clinical setting. This benefit is similar to that obtained in previous clinical trials. The rate for attempting BCS was higher in our study than that in previous multicenter clinical trials.

\section{CONFLICT OF INTEREST}

The authors declare that they have no competing interests.

\section{REFERENCES}

1. Fitzal F, Riedl O, Mittlböck M, Dubsky P, Bartsch R, Steger G, et al. Oncologic safety of breast conserving surgery after tumour downsizing by neoadjuvant therapy: a retrospective single centre cohort study. Breast Cancer Res Treat 2011;127:121-8.

2. Golshan M, Cirrincione CT, Sikov WM, Berry DA, Jasinski S, Weisberg TF, et al. Impact of neoadjuvant chemotherapy in stage IIIII triple negative breast cancer on eligibility for breast-conserving surgery and breast conservation rates: surgical results from CALGB 40603 (Alliance). Ann Surg 2015;262:434-9.

3. Ohsumi S, Shimozuma K, Morita S, Hara F, Takabatake D, Takashima $\mathrm{S}$, et al. Factors associated with health-related quality-of-life in breast cancer survivors: influence of the type of surgery. Jpn J Clin Oncol 2009;39:491-6.

4. Fisher B, Bryant J, Wolmark N, Mamounas E, Brown A, Fisher ER, et al. Effect of preoperative chemotherapy on the outcome of women with operable breast cancer. J Clin Oncol 1998;16:2672-85.

5. van der Hage JA, van de Velde CJ, Julien JP, Tubiana-Hulin M, Vandervelden C, Duchateau L. Preoperative chemotherapy in primary operable breast cancer: results from the European Organization for Research and Treatment of Cancer trial 10902. J Clin Oncol 2001;19:4224-37.

6. Mieog JS, van der Hage JA, van de Velde CJ. Neoadjuvant chemotherapy for operable breast cancer. Br J Surg 2007;94:1189-200.

7. Golshan M, Cirrincione CT, Sikov WM, Carey LA, Berry DA, Overmoyer B, et al. Impact of neoadjuvant therapy on eligibility for and frequency of breast conservation in stage II-III HER2-positive breast cancer: surgical results of CALGB 40601 (Alliance). Breast Cancer Res Treat 2016;160:297-304.

8. Rastogi P, Anderson SJ, Bear HD, Geyer CE, Kahlenberg MS, Robidoux A, et al. Preoperative chemotherapy: updates of National 
Surgical Adjuvant Breast and Bowel Project Protocols B-18 and B-27. J Clin Oncol 2008;26:778-85.

9. van Nes JG, Putter H, Julien JP, Tubiana-Hulin M, van de Vijver M, Bogaerts J, et al. Preoperative chemotherapy is safe in early breast cancer, even after 10 years of follow-up: clinical and translational results from the EORTC trial 10902. Breast Cancer Res Treat 2009; $115: 101-13$
10. Kim MK, Han W, Moon HG, Ahn SK, Kim J, Lee JW, et al. Nomogram for predicting breast conservation after neoadjuvant chemotherapy. Cancer Res Treat 2015;47:197-207.

11. Shin HC, Han W, Moon HG, Im SA, Moon WK, Park IA, et al. Breast-conserving surgery after tumor downstaging by neoadjuvant chemotherapy is oncologically safe for stage III breast cancer patients. Ann Surg Oncol 2013;20:2582-9. 